\title{
DETERMINAÇÃO DA COMPOSIÇÃO MINERAL DE SUBPRODUTOS AGROINDUSTRIAIS UTILIZADOS NA ALIMENTAÇÃO ANIMAL, PELA TÉCNICA DE ATIVAÇÃO NEUTRÔNICA ${ }^{1}$
}

\author{
JOSÉ CLETO DA SILVA FILHO ${ }^{2}$, MARIA JOSÉ AGUIRRE ARMELIN e ALIOMAR GABRIEL DA SILVA ${ }^{4}$
}

\begin{abstract}
RESUMO - O presente trabalho teve como objetivo analisar alguns subprodutos agroindustriais utilizados na alimentação animal e identificar os principais minerais presentes. Amostras de farelos de algodão, arroz, canola, soja e trigo; farinhas de peixe, carne e penas + vísceras; cascas de algodão, arroz, laranja; bagaços de tomate e de laranja foram coletadas em diferentes locais de produção. O método analítico empregado foi a análise por ativação com nêutrons seguida de espectrometria gama. Os níveis de minerais encontrados em todas as amostras, inclusive aqueles considerados tóxicos, tais como As, $\mathrm{Cd}$ e Hg, não excederam os limites máximos permitidos em dietas para animais domésticos. Os valores obtidos foram comparados com os comumente encontrados em forragens.
\end{abstract}

Termos para indexação: metais pesados.

\section{DETERMINATION OF THE MINERAL COMPOSITION IN AGROINDUSTRIAL BY-PRODUCTS USED IN ANIMAL NUTRITION, BY NEUTRON ACTIVATION ANALYSIS}

\begin{abstract}
This study aimed to analyze some agroindustrial by-products used to feed animals in order to identify the presence of the main minerals. Samples of cotton seed meal, rice, canola, soybean and wheat; fish meal, feather meal, meat meal, feather plus viscera meal; rinds of cotton, rice, orange; citrus pulp and tomato residue were collected in different sites of production of the by-products. The method of analysis used was the neutron activation followed by gamma ray spectrometry. The levels of minerals found in all samples were compared with the levels commonly found in forages. The mineral concentration, even those of the toxic elements, as $\mathrm{As}, \mathrm{Cd}$ and $\mathrm{Hg}$, was at low level and did not exceed the maximum tolerable levels for domestic animals.
\end{abstract}

Index terms: heavy metals.

\section{INTRODUÇÃO}

No Brasil, o processamento de produtos agrícolas para a extração de sucos, óleos e molhos para o consumo humano gera uma grande quantidade de subprodutos oriundos do tratamento industrial, tais como sementes, polpas e cascas (Carvalho, 1992). Esses subprodutos representam um grande poten-

\footnotetext{
${ }^{1}$ Aceito para publicação em 8 de junho de 1998.

${ }^{2}$ Químico, Ph.D., DZO, Universidade Federal de Lavras, Caixa Postal 37, CEP 37200-000 Lavras, MG. E-mail: cleto@ufla.br

${ }^{3}$ Química, Ph.D., Instituto de Pesquisas Energéticas e Nucleares, CNEN, Caixa Postal 11049, CEP 05422-970 São Paulo, SP.

${ }^{4}$ Eng. Agr., Ph.D., Embrapa-Centro de Pesquisa de Pecuária do Sudeste (CPPSE), Caixa Postal 339, CEP 13560-970 São Carlos, SP.
}

cial para alimentação animal, principalmente de ruminantes, que possuem capacidade de transformar resíduos de vegetais em nutrientes para sua própria utilização. Toda essa transformação se dá no rúmen, onde, pela ação dos microorganismos ali presentes, ocorre a decomposição da matéria-prima bruta consumida e a síntese de nutrientes assimiláveis pelo organismo.

Como muitas culturas são tratadas com defensivos agrícolas que podem conter contaminantes, entre os quais alguns metais pesados, é necessário identificar a composição desses subprodutos para saber se não representam riscos de contaminação para os animais. Os metais pesados, uma vez ingeridos, poderão se acumular no tecido animal, principalmente no fígado, rins e músculos. Alguns elementos, como arsênio, cádmio e mercúrio são freqüentemente classificados como elementos tóxicos porque suas ativi- 
dades biológicas estão ligadas às reações tóxicas; embora todos os elementos químicos, sejam eles metais ou não, podem ser tóxicos à espécie humana e aos animais se consumidos em quantidades acima da necessidade de cada indivíduo ou por um longo período (Underwood, 1977).

O objetivo do trabalho foi analisar alguns subprodutos utilizados na alimentação animal e identificar os principais minerais presentes.

\section{MATERIAL E MÉTODOS}

Dezenove amostras (massa de aproximadamente $1 \mathrm{~kg}$ ) de farelos de algodão, arroz, canola, soja e trigo; farinhas de peixe, pena, carne e penas + vísceras; cascas de algodão, arroz, laranja e bagaços de tomate foram coletadas em diferentes locais de produção dos subprodutos. Para a irradiação, pesaram-se alíquotas da ordem de $200 \mathrm{mg}$ e transferiram-se para envelopes de polietileno, previamente tratados com $\mathrm{HNO}_{3}$ p.a., 1:5 (v/v) para eliminação de possíveis impurezas. Como os resultados das análises são expressos em peso seco, calculou-se o teor de umidade de cada material. Para isso, pesou-se cerca de $0,5 \mathrm{~g}$ de cada amostra dentro de cadinho de porcelana, previamente tarado. Cada cadinho foi deixado na estufa a $100^{\circ} \mathrm{C}$ por 24 horas. A umidade de cada amostra foi obtida por meio da diferença entre o peso antes e depois da secagem. Esse valor foi utilizado para corrigir as massas de cada alíquota usada na análise.

Os padrões foram preparados a partir de soluções obtidas pela dissolução dos elementos ou de seus compostos espectroscopicamente puros. Alíquotas de 25 ou $50 \mathrm{~mL}$, dependendo da concentração dessas soluções, foram transferidas por meio de micropipetas para papel de filtro Whatman $\mathrm{n}^{\circ} 41$, de aproximadamente $1 \mathrm{~cm}^{2}$ de área. Os padrões preparados apresentaram as seguintes massas: As (11,4 mg); Cd (50,8 mg); Cr (2,4 mg); Co (980 ng); Fe (205 mg); Hg (1,08 mg); K (395 mg); Mo (67,44 mg); $\mathrm{Sb}(18,48 \mathrm{mg}) ; \mathrm{Zn}(24,3 \mathrm{mg})$. A qualidade dos padrões assim preparados foi verificada por meio da análise de materiais de referência.

Grupos de cinco amostras juntamente com os padrões (As, Cd, Cr, Co, Fe, Hg, K, Mo, Na, Sb e Zn) foram irradiados dentro de um recipiente de alumínio sob um fluxo de nêutrons térmicos de aproximadamente $5 \times 10^{12} \mathrm{n} \mathrm{cm}^{-2} \mathrm{~s}^{-1}$ por um período de oito horas, no reator IEA-R1. Após a irradiação, amostras e padrões foram transferidos para recipientes adequados para a medida da radiação gama. $\mathrm{O}$ espectro da radiação gama da amostra foi determinado duas vezes.
Na primeira vez, a amostra foi medida por três horas após um tempo de esfriamento de três a quatro dias, para a medida nos fotopicos correspondentes aos seguintes radionuclídeos: ${ }^{76} \mathrm{As}$ em $559 \mathrm{keV},{ }^{115} \mathrm{Cd}$ em $336 \mathrm{keV},{ }^{42} \mathrm{~K}$ em $1.525 \mathrm{keV},{ }^{99} \mathrm{Mo} \mathrm{em} 140 \mathrm{keV},{ }^{24} \mathrm{Na}$ em $1.368 \mathrm{keV},{ }^{122} \mathrm{Sb}$ em $564 \mathrm{keV}$. Em seguida mediram-se os padrões correspondentes a esses elementos, por 10 minutos cada.

A segunda medida das amostras foi feita depois de um tempo de esfriamento de 10 dias. Nessa etapa, cada amostra foi contada por um tempo mínimo de quatro horas para as medidas dos fotopicos correspondentes à radiação gama do ${ }^{51} \mathrm{Cr}$ em $320 \mathrm{keV},{ }^{60} \mathrm{Co}$ em $1.330 \mathrm{keV},{ }^{59} \mathrm{Fe}$ em $1.098 \mathrm{keV}$, ${ }^{203} \mathrm{Hg}$ em $279 \mathrm{keV}$ e ${ }^{65} \mathrm{Zn}$ em $1.115 \mathrm{keV}$. Uma vez terminadas as contagens, as áreas sob os fotopicos dos radionuclídeos de interesse da amostra foram comparadas com as respectivas áreas sob os fotopicos dos padrões para a determinação das concentrações dos elementos analisados. O equipamento usado para as medidas da radiação gama foi um detector de Ge hiperpuro da EG \& ORTEC, modelo 20195, com resolução de $1,95 \mathrm{keV}$ para o fotopico de $1.332 \mathrm{keV}$ do ${ }^{60} \mathrm{Co}$. Acoplado ao detector, tinha-se um sistema eletrônico constituído de BUFFER - 918 A de 8.000 canais, marca EG \& ORTEC, amplificador, fonte de alta tensão e microcomputador. A função do microcomputador era analisar os dados armazenados na memória do multicanal por meio de um programa em linguagem Turbo Basic.

\section{RESULTADOS E DISCUSSÃO}

A Tabela 1 mostra os resultados da determinação dos elementos nos subprodutos analisados. Os locais onde os subprodutos foram coletados estão identificado por números, e cada resultado é média de duas repetições.

Os valores obtidos de arsênio (As) estão abaixo do permitido em alimentos para animais, que é de 50 ppm (National Research Council, 1980). Apenas a farinha de peixe apresentou uma maior concentração desse metal e em muitas amostras esteve abaixo do limite de detecção do método, nas condições experimentais utilizadas. Níveis elevados desse metal nas plantas podem muitas vezes ser reflexo do seu acúmulo no solo, que em alguns casos chegam a concentrações de até 40 ppm (Underwood, 1977). Porém os subprodutos agroindustriais oriundos do processamento de alimentos para a espécie humana não devem apresentar níveis elevados de As, exceto em caso de contaminação acidental, pois geralmente 
TABELA 1. Resultados da determinação de minerais nos subprodutos (média de duas repetições).

\begin{tabular}{|c|c|c|c|c|c|c|c|c|c|c|c|}
\hline Amostra & Local & As $(\mu \mathrm{g} / \mathrm{g})$ & $\mathrm{Ca}(\%)$ & $\mathrm{Co}(\mu \mathrm{g} / \mathrm{g})$ & $\mathrm{Cr}(\mu \mathrm{g} / \mathrm{g})$ & $\mathrm{Fe}(\mu \mathrm{g} / \mathrm{g})$ & $\mathrm{Hg}(\mu \mathrm{g} / \mathrm{g})$ & $\mathrm{K}(\%)$ & Mo $(\mu \mathrm{g} / \mathrm{g})$ & $\mathrm{Na}(\mu \mathrm{g} / \mathrm{g})$ & $\mathrm{Sb}(\mu \mathrm{g} / \mathrm{g}$ \\
\hline Farelo de algodão & 2 & n. d. ${ }^{1}$ & 0,15 & 0,22 & 0,56 & 149 & 0,131 & 1,65 & 0,56 & 25 & 0.008 \\
\hline Farelo de algodão & 4 & n. d. & 0,11 & 0,19 & n. d. & 101 & 0,053 & 1,45 & 0,65 & 21 & 0,031 \\
\hline Farelo de arroz & 1 & 0,05 & 0,04 & 0,042 & n. d. & 119 & 0,237 & 0,54 & 1,50 & 16 & 0,008 \\
\hline Farelo de soja & 1 & 0,04 & 0,32 & 0,126 & 0,19 & 210 & 0,064 & 2,13 & 4,70 & 6 & 0,007 \\
\hline Farelo de canola & 3 & 0,06 & 0,51 & 0,205 & 0,29 & 329 & 0,267 & 1,53 & 0,80 & 130 & 0,016 \\
\hline Farelo de cevada & 8 & n. d. & 0,28 & 0,032 & 0,56 & 184 & 0,522 & 0,03 & n. d. & 50 & 0,011 \\
\hline Farelo de trigo & 2 & 0,03 & 0,97 & 0,041 & 0,10 & 171 & 0,162 & 1,34 & 2,84 & 50 & - \\
\hline Casca de algodão & 1 & 0,02 & 0,09 & 0,062 & 0,03 & 23 & 0,076 & 1,14 & 0,33 & 30 & 0,016 \\
\hline Casca de arroz & 5 & 0,37 & 0,10 & 0,517 & 0,26 & 53 & n. d. & 0,24 & n. d. & 30 & 0,017 \\
\hline Casca de soja & 1 & 0,12 & 0,29 & 0,184 & 0,38 & 265 & 0,108 & 2,10 & 3,90 & 580 & 0,011 \\
\hline Casca de laranja & 7 & n. d. & 0,63 & 0,018 & 0,15 & 43 & 0,091 & 1,40 & n. d. & 330 & 0,086 \\
\hline Casca de cevada & 8 & n. d. & 0,07 & 0,055 & 0,38 & 142 & 0,462 & 0,88 & n. d. & 170 & - \\
\hline Farinha de carne & 5 & n. d. & 0,06 & 0,04 & 0,36 & 282 & 0,107 & - & n. d. & - & 0,009 \\
\hline Farinha de peixe & 1 & 1,64 & 5,47 & 0,113 & 0,51 & 606 & 0,238 & 0,025 & n. d. & 5430 & - \\
\hline Penas + vísceras & 5 & 1,0 & 1,29 & 0,081 & 0,61 & 527 & 0,238 & 0,15 & n. d. & 960 & 0,021 \\
\hline Bagaço tomate 1 & 6 & n. d. & 0,12 & 0,349 & 0,15 & 92 & 0,159 & 1,10 & n. d. & 120 & 0,007 \\
\hline Bagaço tomate 2 & 6 & n. d. & 0,11 & 0,442 & 0,37 & 452 & 0,200 & 1,40 & n. d. & 230 & 0,004 \\
\hline Bagaço tomate 3 & 6 & n. d. & 0,25 & 0,561 & 3,35 & 915 & 0,226 & 0,96 & n. d. & 130 & 0,013 \\
\hline Bagaço de laranja & 9 & 0,18 & 0,36 & 0,082 & 6,27 & 789 & 0,058 & 0,99 & n. d. & 270 & 0,025 \\
\hline
\end{tabular}


os vegetais apresentam valores abaixo de $0,5 \mathrm{ppm}$ e raramente excedem a $1 \mathrm{ppm}$ no produto fresco (Willians \& McDowell, 1985).

O monitoramento de cádmio $(\mathrm{Cd})$ nos subprodutos é necessário, uma vez que em animais o valor máximo permitido na dieta é de $0,5 \mathrm{ppm}$ (National Research Council, 1980), porém nas condições experimentais de análise não foi possível sua detecção. O limite de detecção desse elemento nas amostras variou de 0,15 a $0,53 \mathrm{ppm}$, o qual foi calculado segundo Keith et al. (1983), que consideram a condição mínima para sua detecção, um nível de confiança de $99 \%$ e que a contagem no fotopico esteja acima da radiação de fundo três vezes o desvio-padrão dessa radiação. Willians \& McDowell (1985) afirmam que nos vegetais, castanhas e frutas, as concentrações de Cd podem variar de 0,04 a 0,08 ppm. Já Teeuwen \& Wreman (1986) ao analisarem amostras de alimentos compostos, usados na alimentação animal, encontraram valores de 0,13 a $0,17 \mathrm{ppm}$. No presente trabalho, a primeira amostragem revelou valores próximos ao valor máximo permitido em farelos de algodão, soja e trigo, mas como há ausência de dados na literatura com tais tipos de amostras que permitam a comparação, é necessário cautela ao considerar os resultados.

Os solos, principalmente aqueles que durante muito tempo receberam fertilizantes cuja fabricação inclui o uso de fosfatos naturais sem tratamento, podem acumular $\mathrm{Cd}$ e posteriormente passar para as plantas (Willians \& David, 1973; Mortvedt et al., 1981), embora Mortvedt (1987) relate que a aplicação de um fertilizante com concentração de $\mathrm{Cd}$ em torno de 10 ppm não aumentou significativamente o nível do elemento nas plantas cultivadas no local.

Quanto ao mercúrio, os valores encontrados em alguns casos são altos, considerando que nas frutas, vegetais e grãos geralmente são detectados níveis de 0,005 a 0,035 ppm (Underwood, 1977). Entretanto, no Brasil, praticamente inexistem dados a respeito que permitam uma comparação com os obtidos no presente trabalho. Porém, uma vez que o nível máximo tolerável de $\mathrm{Hg}$ na dieta de animais é de 2 ppm (National Research Council, 1980), esses dados estão abaixo do permitido e não são preocupantes à primeira vista. Os valores obtidos variaram de 0,053 a $0,267 \mathrm{ppm}$. Apenas a cevada apresentou valores mais elevados $(0,462$ e 0,522 ppm para a casca e farelo). Na Holanda, pesquisadores encontraram níveis de $\mathrm{Hg}$ de até 0,2 ppm nos talos e folhas de beterraba utilizados na alimentação animal (Wriesma et al., 1986).

O cálcio $(\mathrm{Ca})$, por estar presente nas plantas em maior quantidade, dificilmente mostrar-se-á deficiente (Underwood, 1981). De modo geral, a maioria dos trabalhos relatam níveis de Ca de, no mínimo, 0,17\% nas forragens (Possenti et al., 1992). No caso dos subprodutos, é difícil fazer uma comparação com os valores encontrados, pois a maioria deles são resultantes do processamento de grãos, que possuem uma concentração menor de $\mathrm{Ca}$ em sua composição (McDowell, 1985). As amostras que apresentaram os valores mais baixos foram o farelo de arroz, a casca de cevada e a casca de algodão, contendo 0,04 , 0,07 e $0,09 \%$, respectivamente. O mais alto valor foi verificado na farinha de peixe $(5,47 \%)$, mas em função da natureza da amostra é considerado perfeitamente normal.

O zinco é um nutriente importante e essencial no ciclo biológico, sendo vital principalmente para as metaloenzimas (National Research Council, 1980). De modo geral, as forragens e grãos possuem níveis que variam de 20 a $30 \mathrm{ppm}$. O teor de $\mathrm{Zn}$ em leguminosas como soja e amendoim podem apresentar valores de até $70 \mathrm{ppm}$, mas são as farinhas de peixe e de vísceras que apresentam sempre os maiores níveis, geralmente em torno de $100 \mathrm{ppm}$ (Underwood, 1977; National Research Council, 1980). No presente trabalho, o menor valor observado foi de 7 ppm (casca de algodão e polpa cítrica), enquanto o máximo foi de $162 \mathrm{ppm}$ (farinha de peixe), confirmando as afirmações acima. $O$ valor médio observado foi de 60,4 ppm. Conrad et al. (1980) citam valores de 19 a 39 ppm em forragens tropicais. Nas forragens brasileiras os valores não estão muito distantes dos citados acima. Analisando 120 forragens no Estado de Mato Grosso em duas épocas distintas (seca e chuvosa), Sousa et al. (1982) reportaram valores de 28 e 25 ppm, respectivamente.

O molibdênio é outro elemento traço essencial presente nos vegetais. É um componente importante das enzimas responsáveis pela oxidação das purinas e do metabolismo de ferro nos animais. As pastagens contêm sempre concentrações extremamente baixas desse mineral, consideram-se normais aque- 
las de 0,1 a 3 ppm (Underwood, 1977), porém parece não haver na literatura relatos de sintomas de sua deficiência em ruminantes (McDowell, 1985). No caso dos subprodutos analisados, as amostras apresentaram valores de 0,34 a $4,7 \mathrm{ppm}$, sendo os mais elevados observados nos subprodutos da soja (farelo e casca), porém próximos das concentrações normalmente encontradas nas forragens. No Brasil, Sousa et al. (1980), analisando forragens no Estado de Mato Grosso, reportaram concentrações de Mo de 0,1 a 2,2 ppm. Falta, contudo, suporte bibliográfico que possibilite uma comparação entre amostras de subprodutos agroindustriais com os analisados no presente trabalho.

A presença de cromo $(\mathrm{Cr})$ nas plantas, mesmo em baixos níveis, é suficiente para suprir os requerimentos dos animais. De acordo com Underwood (1977), a maioria dos alimentos traz em sua composição níveis de 0,2 a $0,8 \mathrm{ppm}$, porém com o advento de novas técnicas de análise é possível a detecção de níveis mais baixos com precisão. Esse elemento é importante do ponto de vista nutricional por funcionar como um cofator na ativação da insulina em mamíferos, sendo também o agente responsável pelo fator de tolerância à glicose, requerido na manutenção do metabolismo desta (McDowell, 1985).

Os níveis de $\mathrm{Cr}$ encontrados nas amostras neste estudo estão em concordância com os relatados na literatura, exceto quanto aos dos bagaços de tomate e de laranja, que apresentaram valores de 3,35 e 6,27 ppm, respectivamente. As demais amostras tiveram concentrações variando de 0,03 a $0,61 \mathrm{ppm}$. McDowell (1985) cita valores de 0,11, 0,24, 0,424 e 1,17 ppm, respectivamente em milho, germe de trigo, farelo de trigo e levedura de cerveja. Fávaro et al. (1994) analisando folhas de citrus, detectaram nível médio de $\mathrm{Cr}$ de 1,0 ppm. Os mesmos autores analisaram a dieta total consumida por várias populações humanas em diferentes regiões do Brasil e reportaram um valor médio de $0,5 \mathrm{ppm}$. A literatura, de modo geral, não registra casos de deficiência de $\mathrm{Cr}$ em ruminantes.

Detectou-se antimônio em praticamente todas as amostras, cujos valores variaram de 0,080 a $0,086 \mathrm{ppm}$. Esse elemento não possui nenhuma função nos organismos vivos e também não figura entre os considerados tóxicos (Underwood, 1977). Talvez por isso poucos têm sido os trabalhos publicados relatando a sua presença em alimentos e forrageiras. Sua propriedade tóxica está sempre relacionada à atividade industrial, principalmente em locais onde há mineração, uso indevido de recipientes para guardar alimentos, produção de ligas metálicas e manufatura de abrasivos. Casos de intoxicação em animais têm sido relatados apenas experimentalmente (National Research Council, 1980).

Os níveis de ferro determinados apresentaram-se com bastante variação, mas, de acordo com o tipo de amostra, assemelham-se aos considerados normais na literatura. As cascas de algodão, laranja e arroz apresentaram os valores mais baixos $(23,43$ e 53 ppm, respectivamente). Os níveis de Fe encontrados nas plantas são basicamente determinados pela espécie e tipo de solo no qual se encontra a espécie vegetal. As plantas cultivadas podem apresentar níveis de 100 a 700 ppm de Fe em sua composição (National Research Council, 1980), e dados de Conrad et al. (1980) reforçam essa afirmativa ao relatarem teores de Fe de 487 a 553 ppm em forragens nativas no Estado de Mato Grosso.

A presença de cobalto nos subprodutos se deu em níveis aceitáveis, variando de 0,018 a 0,56 ppm, embora em amostras diferentes. A literatura destaca que numa pastagem considerada de boa qualidade há concentração de, no mínimo, 0,1 ppm desse mineral, enquanto em áreas deficientes têm sido reportados valores que vão de 0,004 a 0,07 ppm (McDowell, 1985). O requerimento de Co pelos ruminantes se baseia na necessidade que os microorganismos do rúmen têm desse elemento para a síntese de vitamina $\mathrm{B}_{12}$, que é usada pelo hospedeiro.

Os teores de sódio $(\mathrm{Na})$ nas plantas de modo geral são insuficientes para suprir a necessidade dos ruminantes. As forrageiras e leguminosas trazem em sua composição valores baixos desse mineral. De acordo com o National Research Council (1980), temse em alguns produtos vegetais as concentrações médias de Na: 40 ppm no milho; 80 ppm no feno de timóteo e na aveia; 200 ppm na soja; 700 ppm no feno de alfafa; e até 1.400 ppm no feno de trevo. As concentrações aqui obtidas nos subprodutos de origem vegetal também foram baixas, não diferindo em quase nada das concentrações relatadas anteriormente. Da mesma forma, dados muito próximos foram reportados por Conrad et al. (1980) em forragens do Esta- 
do de Mato Grosso, de 84 a 194 ppm, bem como por Sousa et al. (1982).

Os valores encontrados de potássio $(\mathrm{K})$ variaram de 0,029 a $2,13 \%$ e estão de acordo com os níveis normalmente reportados em forragens e grãos. Os grãos, de um modo geral, contêm menos de $0,5 \%$ desse mineral. Nas forragens em crescimento, os níveis de $\mathrm{K}$ são mais elevados ( $1 \mathrm{a} 4 \%$ ), e à medida que as plantas tornam-se maduras eles tendem a baixar (McDowell, 1985). Sousa et al. (1982) reportaram níveis de 0,82 e 1,93\% de $\mathrm{K}$ em forrageiras nas épocas seca e chuvosa, respectivamente. O National Research Council (1980) estipulou o nível máximo de ingestão de $\mathrm{K}$ em $3 \%$, mas dificilmente há intoxicação por tal elemento, pois mesmo numa pastagem nova com níveis elevados, o que resulta em maior ingestão pelos animais, o consumo mesmo acima do requerido será rapidamente excretado. Altos níveis de $\mathrm{K}$ podem interferir no metabolismo de magnésio no organismo animal (McDowell, 1985).

\section{CONCLUSÕES}

1. Com relação aos minerais presentes, as concentrações encontram-se em níveis aceitáveis de acordo com a literatura pertinente.

2. A presença de arsênio e mercúrio nos subprodutos analisados não excede os limites permitidos em dietas de animais domésticos.

\section{REFERÊNCIAS}

CARVALHO, F.C. Disponibilidade de resíduos agroindustriais e do beneficiamento de produtos agrícolas. In: SIMPÓSIO SOBRE UTILIZAÇÃO DE SUBPRODUTOS AGROINDUSTRIAIS E RESÍDUOS DE COLHEITA NA ALIMENTAÇÃO DE RUMINANTES, São Carlos, 1992. Anais... São Carlos: Embrapa-UEPAE São Carlos, 1992. p.7-27.

CONRAD, J.H.; SOUSA, J.C.; MENDES, M.O.; BLUE, W.G.; McDOWELL, L.R. Iron, manganese, sodium and zinc interrelationship in a tropical soil, plant and animal system. In: VERDE, L.S.; FERNANDEZ, A. (Eds.). IV World Conference on Animal Production, Buenos Aires: [s.n.], 1980. p.48-53. Separata da IV Conference on Animal Production, 1978, Buenos Aires.
FÁVARO, D.I.T.; MAIHARA, V.A.; ARMELIN, M.J.A.; VASCONCELOS, M.B.A. Determination of As, Cd, $\mathrm{Cr}, \mathrm{Cu}, \mathrm{Hg}, \mathrm{Sb}$ and $\mathrm{Se}$ concentrations by radiochemical neutron activation analysis in different Brazilian regional diets. Journal of Radioanalytical and Nuclear Chemistry, v.181, n.2, p.385-394, 1994.

KEITH, L.H.; CRUMMET, W.; DEGAN JUNIOR, J.; LIBBY, R.A.; TAYLOR, J.K.; WENTLER, G. Principles of environmental analysis. Analytical Chemistry, v.55, p.2210-2218, 1983.

McDOWELL, L.R. Nutrition of grazing ruminants in warm climates, Orlando: Academic Press, 1985. $443 p$.

MORTVEDT, J.J. Cadmium levels in soils and plants from some long-term soil fertility experiments in the United States of America. Journal of Environmental Quality, Madison, v.16, n.2, p.137-142, 1987.

MORTVEDT, J.J.; MAYS, D.A.; OSBORN, G. Uptake by wheat of cadmium and other heavy metal contaminants in phosphate fertilizer. Journal of Environmental Quality, Madison, v.10, n.2, p.193-197, 1981.

NATIONAL RESEARCH COUNCIL. (Washington, DC). Mineral tolerance of domestic animals. Washington, DC: National Academic Press, 1980. 577p.

POSSENTI, R.A.; LOBÃO, A.O.; RIBEIRO, W.R.R.; DELISTOIANOV, J. Determinações de minerais em forragens e tecidos de bovinos. Boletim da Indústria Animal, Nova Odessa, v.49, n.2, p.131-144, 1992.

SOUSA, J.C. de; CONRAD, J.H.; McDOWELL, L.R.; AMMERMAN, C.B.; BLUE, W.G. Inter-relações entre minerais no solo, forrageiras e tecido animal. 2. Cobre e molibdênio. Pesquisa Agropecuária Brasileira, Brasília, v.15, n.3, p.335-341, 1980.

SOUSA, J.C. de; CONRAD, J.H.; MOTT, G.O.; McDOWELL, L.R.; AMMERMAN, C.B.; BLUE, W.G. Inter-relações entre minerais no solo, plantas forrageiras e tecido animal no norte de Mato Grosso. 4. Zinco, magnésio, sódio e potássio. Pesquisa Agropecuária Brasileira, Brasília, v.17, n.1, p.11-20, 1982.

TEEUWEN, J.J.M.H.G. vos; WREMAN, K. Cadmium and lead in compounded feeds. Netherlands Journal of Agricultural Science, Wageningen, v.34, p.437-440, 1986. 
UNDERWOOD, E.J. Sources of minerals. In: UNDERWOOD, E.J. The mineral nutrition of livestock. 2.ed. Farnhan Royal: CAB, 1981. ch.2, p.9-19.

UNDERWOOD, E.J. Trace elements in human and animal nutrition. 4.ed. London: Academic Press, 1977. $545 \mathrm{p}$.

WILLIANS, C.H.; DAVID, D.J. The effects of superphosphate on the cadmium content of soils and plants. Australian Journal of Soil Research, Melbourne, v.11, p.43-56, 1973.
WILLIANS, S.N.; McDOWELL, L.R. Newly discovered and toxic elements. In: McDOWELL, L.R. (Ed.). Nutrition of grazing ruminants in warm climates. Orlando: Academic Press, 1985. ch.14, p.317-338.

WRIESMA, D.; VAN GOOR BEREND, J.; VAN DER VEEN NICOLAAS, G. Cadmium, lead, mercury and arsenic concentrations in crops and corresponding soils in the Netherlands. Journal of Agriculture and Food Chemistry, Easton, v.34, p.1067-1074, 1986. 